\title{
Parte VI. Tratamiento antiparasitario de la enfermedad de Chagas
}

\author{
Comité de Parasitología, Departamento de Enfermedades Emergentes y Re-emergentes, \\ Ministerio de Salud de Chile*
}

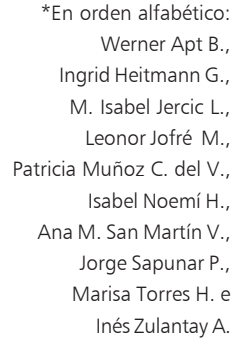

\section{Part VI. Antiparasitic treatment for Chagas disease}

As expert consensus has been arisen about universal antiparasitic treatment for all patients infected with Trypanosoma cruzi, most important drugs licensed for Chagas disease treatment are reviewed: nifurtimox and benznidazol, their mechanisms of action, doses, treatment schedules, adverse effects and contraindications. Two other drugs used for Chagas disease treatment, for which a Chilean experience may be exhibited, are allopurinol and itraconazole. Indications for treatment of Chagas disease in immunocompetent patients and inmunocompromised hosts are detailed. This chapter refers besides to the evaluation and monitoring of antiparasitic therapy in inmunocompromised patients, the availability of drugs and includes various forms facsimiles suggested to perform clinical and laboratory follow up of patients that undergo treatment, indicating the prescribed drug, adverse effects and time of follow up.

Key words: Chagas disease, American Trypanosomiasis, treatment.

Palabras clave: Enfermedad de Chagas, tripanosomiasis americana, tratamiento.
$\mathrm{T}$ odo paciente chagásico debe ser tratado, a excepción de los enfermos crónicos terminales. Cada caso debe ser evaluado en relación al costo-beneficio de la terapia antiparasitaria específica. Los fármacos tripanomicidas clásicos aprobados hasta ahora para el tratamiento de la enfermedad de Chagas son: nifurtimox (NFX) y benznidazol (BNZ), aunque se han incorporado otras alternativas, en especial en adultos en fase crónica indeterminada y determinada (ver nota de la editora).

Aparte de la adecuada administración del tratamiento, deben monitorizarse sus efectos adversos y su efecto parasiticida. Para estos últimos, existen métodos como el de Strout, RPC cuantitativa y cualitativa para $T$. cruzi y, aunque muy tardío en sus resultados, en casos debidamente justificados, el xenodiagnóstico.

\section{Fármacos antichagásicos}

Nifurtimox. Lampit®, Laboratorio Bayer, es un análogo de nitrofuranos. Tiene efecto tripanomicida, actúa contra las formas amastigote y tripamastigote de $T$. cruzi. Fue aprobado su uso en enfermedad de Chagas en el año 1965. Ha demostrado ser efectivo en las fases aguda, crónica indeterminada y crónica determinada de la enfermedad, con una cura parasitológica de $76 \%$ en la etapa aguda y porcentaje variable en la etapa crónica, habiendo resultados contradictorios en algu- nas series. La acción de este medicamento está relacionada con la generación de productos de la reducción de oxígeno, contra los cuales el tripanosoma es deficiente en mecanismos de detoxificación, lo que lo hace susceptible al estrés oxidativo.

Los efectos secundarios se presentan en $30 \%$ de los casos, especialmente en adultos. Puede producir anorexia, pérdida de peso, manifestaciones gastrointestinales como náuseas, vómitos, dolor abdominal, diarrea, dermatitis y compromiso del SNC con insomnio, alucinaciones, parestesias y psicosis. Las reacciones de toxicidad y los efectos colaterales debidos a BNZ y a NFX pueden ser importantes. Nifurtimox provoca más comúnmente manifestaciones del aparato digestivo tales como epigastralgia, anorexia, náuseas, vómitos y pérdida de peso. Está contraindicado en mujeres embarazadas y en pacientes con insuficiencia renal y hepática.

Benznidazol. Radanil ${ }^{\circledR}$, Argentina, Rochagan $($, Brasil, (Laboratorio Roche). Es también un fármaco tripanomicida. Actúa uniéndose en forma covalente a los intermediarios de la nitrorreducción con los componentes del parásito, ADN, lípidos y proteínas. Es eficaz en el tratamiento de la fase aguda, en la fase crónica indeterminada y en la crónica determinada, como se ha demostrado en estudios realizados en niños en Brasil y Argentina.

Los efectos adversos se dividen en tres tipos: 
- Dermatológicos: erupción cutánea que aparece entre los 7-10 días de tratamiento, edema generalizado, fiebre, adenopatías, mialgia y artralgia.

- Hematológicos: depresión de la médula ósea con trombocitopenia, púrpura y agranulocitosis, que es la manifestación más grave.

- Compromiso neurológico: polineuropatía, parestesia y polineuritis periférica.

En animales de experimentación se ha demostrado un efecto mutagénico y teratogénico, lo que no se ha evidenciado en el hombre. Está contraindicado en mujeres embarazadas y en pacientes con insuficiencia hepática y renal.

Allopurinol. Inhibidor de la síntesis de purinas. No es eficaz en el tratamiento de la fase aguda. Estudios en pacientes crónicos, en los cuales se comparó allopurinol con BNZ y NFX, mostraron una negativización de $45-92 \%$ de la serología $T$. cruzi, con menos efectos adversos en el grupo tratado con allopurinol. Se ha usado en pacientes sometidos a trasplante cardíaco con buena tolerancia. No se ha empleado en niños.

Itraconazol. Es un derivado sintético del imidazol. Estudios realizados en adultos demostraron la curación parasitológica en $20 \%$ de los casos, con $50 \%$ de mejoría de las alteraciones electrocardiográficas. Debe ser usado por períodos prolongados. Produce reacciones adversas de tipo idiopáticas, como la insuficiencia hepática, las que deben ser monitorizadas.

\section{Indicación de tratamiento de la enfermedad de Chagas en pacientes inmunocompetentes}

Chagas agudo. Debe tratarse siempre, para prevenir complicaciones y la evolución a la fase crónica. Es en esta fase donde se ha demostrado la mayor eficacia de NFX y BNZ.

Nifurtimox se utiliza en dosis de $8 \mathrm{mg} / \mathrm{kg} / \mathrm{dí} a$, fraccionado cada 8 horas, durante 60 a 90 días. En niños se usa en dosis de $10 \mathrm{mg} / \mathrm{kg} /$ día, por el mismo período.

Benznidazol se usa en dosis de $5 \mathrm{mg} / \mathrm{kg} /$ día en dos dosis, durante 60 días en adultos. En niños bajo $40 \mathrm{~kg}$ de peso se utiliza $5-10 \mathrm{mg} / \mathrm{kg} /$ día, fraccionado en dos dosis, durante 30 días. En lactantes se utilizan dosis de $10 \mathrm{mg} / \mathrm{kg} /$ día, fraccionado cada 12 horas.

Chagas congénito. Se deben tratar todos los casos pues se ha visto una negativización de la serología y la parasitemia en $80 \%$ de los pacientes, lográndose una mejor respuesta mientras más precoz se inicie el tratamiento. Se debe usar NFX en dosis de $8-10 \mathrm{mg} / \mathrm{kg} /$ día durante 60 días, fraccionado cada 8 ó 12 horas y benznidazol en dosis de $5-7 \mathrm{mg} / \mathrm{kg} /$ día, durante 30 días. Para evitar efectos en el SNC se recomienda asociar a fenobarbital en dosis terapéuticas los prime- ros quince días de tratamiento. En el caso de reacciones dermatológicas secundarias, se sugiere asociar antihistamínicos al tratamiento específico. Las reacciones adversas en el RN, son de menor cuantía comparadas con la de adultos

Es importante hacer el diagnóstico precoz de los casos congénitos para tratarlos oportunamente, por lo cual se sugiere realizar en:

- La mujer embarazada: pesquisa serológica para la infección por T cruzi junto con el VDRL en el primer trimestre del embarazo y un seguimiento de los casos positivos, hasta confirmar o descartar el diagnóstico de infección congénita. Esta actividad debe realizarse en toda mujer en edad fértil o en mujeres gestantes de zonas endémicas y en mujeres con el antecedente de haber vivido en ellas.

- El recién nacido: pesquisa serológica para la infección por T cruzi junto con el estudio de VDRL. En los casos positivos se debe hacer un seguimiento hasta confirmar o descartar el diagnóstico.

\section{Chagas en pediatría (lactantes, pre-escolares, escolares y adolescentes)}

El éxito del tratamiento va a depender de la precocidad de su inicio. Estudios randomizados, doble ciego, han comprobado en escolares tratados con BNZ, un éxito terapéutico de 55,8 y $60 \%$, respectivamente.

\section{Chagas accidental o por vía parenteral}

En personas que trabajan en laboratorios y que accidentalmente presentan un accidente cortopunzante por muestras contaminadas con formas infectantes de T. cruzi, se debe confirmar la contaminación del objeto con el parásito y la infección posterior del paciente (mediante serología y RPC). Si son positivas, se debe iniciar tratamiento en forma inmediata, con BNZ en dosis de 7-10 mg/kg/día, al menos durante 15 días, dependiendo de su estado inmunológico. Debe realizarse estudio serológico a los 15, 30 y 60 días del ocurrido el accidente.

\section{Fase crónica indeterminada}

En una revisión sistemática de Cochrane se encontró que los derivados nitroimidazólicos modifican la evolución, comparándolos con población infectada que recibió placebo y otros agentes, por lo que se debe tratar en esta fase con fármacos antiparasitarios durante 2 a 3 meses, dependiendo del medicamento utilizado y el caso en particular.

\section{Fase crónica determinada}

Un meta-análisis de Cochrane no encontró evidencia suficiente a favor del uso de NFX y BNZ en el tratamiento de la cardiopatía chagásica grave. 


\section{Indicaciones para efectuar tratamiento de la enfermedad de Chagas en pacientes inmunocomprometidos}

Se debe iniciar el tratamiento antiparasitario en forma inmediata, ya sea en fase aguda o en reactivación, a fin de detener la multiplicación parasitaria y la diseminación tisular del parásito.

Se emplean los antiparasitarios clásicos, en dosis habituales, hasta que se logre reconstituir la respuesta inmune del hospedero (en algunos casos 60 días o más) Una vez normalizada la alteración del sistema inmune, entre ellos la relación CD4/CD8, se cambia el esquema antiparasitario de dosis terapéuticas a día por medio, balanceando los efectos parasiticidas con los efectos adversos. Los pacientes con SIDA, sin terapia anti-retroviral, son los más gravemente afectados. En ellos, una vez normalizados los niveles de CD4 con terapia específica antiviral, se puede emplear esquemas de mantención.

En el caso de pacientes sometidos a trasplante de precursores hematopoyéticos, portadores de infección por T. cruzi, (los cuales recidivan hasta en $40 \%$ de los casos), el tratamiento se mantiene por dos años. En los receptores de órganos sólidos, debe tratarse por el período en que se utilicen los inmunosupresores y que tengan niveles de CD4 bajos para montar una adecuada respuesta inmune.

En estos pacientes, los fármacos más usados son los tradicionales, NFX y BNZ. De no responder al tratamiento habitual, se sugiere consultar a los expertos. En el modelo de pacientes expuestos a trasplante de órganos sólidos se pueden producir diversas situaciones, tales como primoinfecciones y reactivaciones, por lo cual es importante hacer un buen tamizaje donante-receptor, previo al trasplante.

Las manifestaciones clínicas de la reactivación difieren habitualmente de las de fase aguda (primoinfección), por lo cual es muy relevante el monitoreo de los pacientes post transplante. En ambas situaciones, el receptor que recibe un órgano de donante chagásico o bien un receptor chagásico reactivado, se puede indicar terapia con NFX o BNZ.

Además, medicamentos como NFX, BNZ y allopurinol, son eficaces para negativizar parcialmente las parasitemias, en aproximadamente $70 \%$ de los casos. Con ello

- Se previene la incidencia de cardiopatía o de alteraciones electrocardiográficas en aquellas personas infectadas. La incidencia encontrada es de 4,8\%, los que presentan una mayor frecuencia de estas alteraciones.

- Se evita la progresión de la cardiopatía en pacientes con cardiopatías, leves y moderadas
- Disminuye la incidencia de complicaciones cardiológicas y se reduce la mortalidad atribuible a esta infección parasitaria.

Todo lo anteriormente expuesto sería susceptible de ser obviado con la intervención terapéutica.

Tanto BNZ como NFX se presentan en tabletas y las dosis totales diarias deben administrarse en dos o tres fracciones similares, con intervalos de 8 a 10 horas, de preferencia después de ingerir alimentos, para evitar o paliar los efectos adversos que estos fármacos pueden producir.

\section{Evaluación y monitorización de la terapia antiparasitaria en hospederos inmunocomprometidos}

El principal inconveniente en el tratamiento de la enfermedad de Chagas es su larga duración. El tratamiento debe mantenerse por al menos 60 días y el criterio de curación depende de varios factores. Algunos autores consideran necesaria la conversión serológica, pero esto puede suceder excepcionalmente, o bien después de 20 o más años de finalizada, pudiendo existir casos en que el paciente fallezca antes de la seroconversión. Otros consideran como criterio de curación, durante la etapa crónica, a los siguientes parámetros: la conversión del xenodiagnóstico de $(+)$ a (-); conversión de la RPC cuantitativa de (+) a (-) y en los pacientes con cardiopatías, la desaparición de las alteraciones electrocardiográficas, siempre y cuando estos cambios sean permanentes y al menos persistan por doce o más años, independientemente de los resultados de la serología convencional.

En las fases aguda, crónica indeterminada y crónica determinada reciente, se debe efectuar un seguimiento con cultivo de $T$. cruzi en sangre, RPC cuantitativa para T. cruzi, hemograma, perfil bioquímico y/o xenodiagnóstico. Debe recordarse que las pruebas serológicas en pacientes inmunocomprometidos graves, frecuentemente resultan negativas y no sirven para monitorizar los tratamientos. Una negatividad prolongada y persistente de la RPC con las características mencionadas para $T$. cruzi, es considerada como criterio de curación. Algunos autores valoran la importancia de la desaparición de anticuerpos líticos, como un criterio de mejoría complementario.

\section{Disponibilidad de fármacos}

Una situación preocupante son los escasos recursos terapéuticos y la disponibilidad limitada que tienen. Este problema se presenta tanto en nuestro país como en la mayoría de los países endémicos. Por este 
motivo, es necesario educar a la población para que consulte precozmente y evite la automedicación. La Central de Abastecimiento dispone de un stock de fármacos para la red del sistema de salud (*Nota de la editora).

\section{Resumen}

Con el consenso de expertos de que todo paciente infectado con Trypanosoma cruzi debiera recibir tratamiento anti-parasitario, se revisan los principales medicamentos aprobados para la enfermedad de Chagas: nifurtimox y benznidazol, sus mecanismos de acción, dosis, duración del tratamiento, efectos adversos y contraindicaciones. Se mencionan otros dos medicamentos utilizados en el tratamiento, en el que existe alguna experiencia nacional, como son allopurinol e itraconazol. Se revisan las indicaciones de tratamiento de la enfermedad de Chagas en personas inmunocompetentes y las indicaciones de tratamiento en hospederos inmunodeprimidos. Este capítulo finaliza abordando la evaluación y monitorización de la terapia antiparasitaria en inmunodeprimidos, la disponibilidad de medicamentos e incluye facsímiles de formularios sugeridos para realizar el seguimiento clínico y de laboratorio de los pacientes que son sometidos a tratamiento, indicando el fármaco utilizado, los efectos adversos y el tiempo de seguimiento.

\section{Referencias}

1.- Apt W, Aguilera X, Arribada A, Pérez C, Miranda C, Sánchez G, et al. Treatment of chronic Chagas's disease with itraconazole and allopurinol. Am J Trop Med Hyg 1998; 59: 133-8.

2.- Apt W, Arribada A, Zulantay I, Solari I, Sánchez G, Mundana K, et al. Itraconazole or allopurinol in the treatment of chronic american trypanosomiasis. The results of clinical and parasitological examinations 11 years post treatment. Ann Trop Med Parasitol 2005; 99: 733-41.

3.- Apt W. Tratamiento de la enfermedad de Chagas. Parasitol Día 1999; 23.

4.- Buckner F, Wilson A, White T C, Van Voohis W C. Induction of resistance to azole drugs in Trypanosoma cruzi. Antimicrob Agents Chemother 1998; 42: 3245-50.

5.- Ferreira M S, Nishioska S, Silvestre M T,
Vorges A S, Nunes Araujo F R, Rocha A. Reactivation of Chagas disease in patients with AIDS: Report of three new cases and review of the literature. Clin Infect Dis 1997; 25: 1397-400.

6.- Fragata F A, Luquetti A, Prata A, Andrade A, Rassi A, Gontijo E et al. Etiological treatment of Chagas disease. Parasitol Today 1997; 12: 127-30.

7.- Organización Panamericana de la Salud/ Organización Mundial de la Salud. Tratamiento etiológico de la enfermedad de Chagas. Conclusiones de una consulta Técnica Fundación Oswaldo Cruz, Río de Janeiro 23-25 de abril 1998.

8.- Reyes P A, Vallejo M. Trypanocidal drugs for late stage, symptomatic Chagas disease (Trypanosoma cruzi infection). Cochrane Database 2005 Oct 19; (4): 004102. Review.

9.- Rodríguez Soura J, De Castro S L. A critical review on Chagas disease chemotherapy. Mem Inst Oswaldo Cruz 2002; 97: 3-24.
10.- Rodríguez-Morales A J. Nuevas perspectivas en el manejo terapéutico de la enfermedad de Chagas. Rev Peru Med Exp Salud Pública 2005; 22: 113-33.

11.- Schenone H, Contreras M, Solari A, García A, Rojas A, Lorca M. Tratamiento con nifurtimox en niños con infección chagásica. Rev Méd Chile 2003; 131: 1089-90.

12.- Solari A, Saavedra H, Sepúlveda C, Oddo D, Acuña G, Labarca J. et al. Successful treatment of Trypanosoma cruzi encephalitis in a patients with hemophilia and AIDS Clin Infect Dis 1993; 16: 255-9.

13.- Urbina J A. Specific treatment of Chagas disease: current status and new developments. Curr Opin Infect Dis 2001; 14: $733-41$

14.- Villar J C, Marin-Neto J A, Ebrahim S, Yusuf S. Trypanocidal drugs for chronic asymptomatic Trypanosoma cruzi infection. Cochrane Database Syst Rev 2002 (1): CD 003463. Review.

\section{Nota de la Editora}

La disponibilidad de los dos antiparasitarios aprobados para el tratamiento de la infección por Trypanosoma cruzi: nifurtimox y benznidazol, ha sido muy limitada, tanto en Chile como en el resto de Latinoamérica. Las razones que han explicado esta situación son diversas destacando su escasa comercialización por el concepto muy difundido de que la enfermedad de Chagas no tenía tratamiento debido a la poca claridad en establecer los criterios de cura parasitológica. Desde que existe el consenso, cada vez más creciente, de que el daño de la etapa crónica está directamente relacionado con la presencia del parásito y que éste se puede eliminar persistiendo los anticuerpos positivos debido a una memoria inmunológica, que han aumentado los casos debido a una notificación obligatoria y al tamizaje para $T$. cruzi en los bancos de sangre, esta enfermedad ha sido considerada re-emergente en nuestro país.

Por una iniciativa de la OPS, Laboratorio Bayer S.A. se comprometió a reponer en el mercado nuevamente el fármaco nifurtimox.
Este hecho se concretó en nuestro país a contar del mes de julio de 2008, encontrándose disponible en la CENABAST (Central Nacional de Abastecimiento) para los pacientes del sistema público y, a contar del mes de octubre, directamente en el laboratorio, para pacientes privados. La licencia del otro medicamento, benznidazol, de Laboratorio Roche fue vendida a un laboratorio de Pernambuco en Brasil quién será el encargado de su comercialización, por lo que no se encuentra disponible actualmente en Chile. 
Parasitología

\section{Facsímil de formulario para el tratamiento y seguimiento de la enfermedad de Chagas}

Servicio de Salud:

Establecimiento:

Nombre paciente

Rut

Edad

Dirección

Teléfono

Establecimiento de procedencia

Etapa de la infección por Trypanosoma cruzi

Exámenes indirectos $(+)$

Señale método, título de anticuerpos y fecha pre- tratamiento

Exámenes directos:

Indique método, resultado y fecha pre-tratamiento

Fármaco anti-chagásico utilizado

Dosis utilizada y duración

Fecha de inicio tratamiento

Fecha de término tratamiento

Efectos adversos (describa por sistemas y a los cuántos días de tratamiento aparecieron):

piel: dermatitis, vesículas, edema, prurito, rush, púrpura

gastrointestinales: anorexia, náuseas, vómitos, dolores, diarrea

hematológicos: trombocitopenia, anemia, leucopenia, agranulocitosis

SNC: cefalea, insomnio, alucinaciones, polineuropatía, parestesias, polineuritis periférica

Generales: baja de peso, fiebre, mialgias, artralgias, adenopatías

Otros

Nombre de médico tratante

Dirección y teléfono

Correo electrónico

Si por cualquier motivo se cambia el fármaco antichagásico, se debe iniciar otra ficha 


\section{Facsímil de formulario para el seguimiento del tratamiento de pacientes con enfermedad de Chagas}

Servicio de Salud:

Establecimiento:

Nombre del paciente:

Fármaco y dosis utilizada:

\begin{tabular}{|c|c|c|c|c|c|}
\hline Exámenes & Pre- tratamiento & $\begin{array}{l}3 \text { meses después } \\
\text { de tratamiento }\end{array}$ & $\begin{array}{l}6 \text { meses después } \\
\text { de tratamiento }\end{array}$ & $\begin{array}{l}1 \text { año después } \\
\text { de tratamiento }\end{array}$ & $\begin{array}{l}2 \text { años después } \\
\text { de tratamiento }\end{array}$ \\
\hline $\begin{array}{l}\text { Indirectos para enfer- } \\
\text { medad de Chagas (mé } \\
\text { todo y título de Acs.) }\end{array}$ & & & & & \\
\hline $\begin{array}{l}\text { Directos para } \\
\text { Trypanosoma cruzi } \\
\text { Método }\end{array}$ & & & & & \\
\hline $\begin{array}{l}\text { E.C.G: describa lo } \\
\text { encontrado }\end{array}$ & & & & & \\
\hline $\begin{array}{l}\text { Ecocardiograma } \\
\text { (si lo tiene) }\end{array}$ & & & & & \\
\hline $\begin{array}{l}\text { Enema baritada } \\
\text { (si procede) }\end{array}$ & & & & & \\
\hline $\begin{array}{l}\text { Estudio esofágico } \\
\text { (si procede) }\end{array}$ & & & & & \\
\hline $\begin{array}{l}\text { Otros (Ej: Rx tórax, } \\
\text { LCR, fondo de ojos } \\
\text { en casos congénitos) }\end{array}$ & & & & & \\
\hline Otros & & & & & \\
\hline
\end{tabular}

Facsímil para el seguimiento del tratamiento paciente con enfermedad de Chagas

Servicio de Salud:

Establecimiento:

Nombre:

RUT:

Fármaco y dosis utilizada:

\begin{tabular}{|c|c|c|c|c|c|c|}
\hline Exámenes durante el tratamiento & 15 días & 30 días & 45 días & 60 días & 75 días & 90 días \\
\hline \multicolumn{7}{|l|}{ Hemograma, VHS y recuento de plaquetas } \\
\hline \multicolumn{7}{|l|}{ Pruebas de función hepática } \\
\hline \multicolumn{7}{|l|}{ Perfil bioquímico } \\
\hline $\begin{array}{l}\text { Examen físico/químico y sedimento } \\
\text { de orina }\end{array}$ & & & & & & \\
\hline
\end{tabular}

